\title{
The cutaneous contribution to adaptive precision grip
}

\author{
Alice G. Witney ${ }^{1}$, Alan Wing ${ }^{2}$, Jean-Louis Thonnard ${ }^{3}$ and Allan M. Smith ${ }^{4}$ \\ ${ }^{1}$ Department of Zoology, University of Cambridge, Downing Street, Cambridge CB2 3EJ, UK \\ ${ }^{2}$ Sensorimotor Neuroscience (SyMoN), Behavioural Brain Sciences, School of Psychology, University of Birmingham, \\ Birmingham B15 2TT, UK \\ ${ }^{3}$ Unité de Réadaptation et de Médecine Physique, Université Catholique de Louvain, 53 Avenue Mounier, Tour Pasteur (5375), \\ 1200 Brussels, Belgium \\ ${ }^{4}$ Centre de Recherche en Sciences Neurologiques, Département de Physiologie, Université de Montréal, Montréal, Québec, \\ Canada H3T 1J4
}

\begin{abstract}
Only after injury, or perhaps prolonged exposure to cold that is sufficient to numb the fingers, do we suddenly appreciate the complex neural mechanisms that underlie our effortless dexterity in manipulating objects. The nervous system is capable of adapting grip forces to a wide range of object shapes, weights and frictional properties, to provide optimal and secure handling in a variety of potentially perturbing environments. The dynamic interplay between sensory information and motor commands provides the basis for this flexibility, and recent studies supply somewhat unexpected evidence of the essential role played by cutaneous feedback in maintaining and acquiring predictive grip force control. These examples also offer new insights into the adaptive control of other voluntary movements.
\end{abstract}

It is probably fair to say that if finger movements were controlled purely on the basis of sensory feedback, they would be too slow and clumsy to be of much use. A child struggling to tie his shoelaces illustrates our dependence on feedback during the early stages of skill acquisition. As Lashley pointed out many years ago, skilled musicians perform finger movements that are too rapid to be sequentially triggered through feedback [1]. Engineering control theorists have long known that rapid adaptability requires some means of anticipating changing conditions rather than reacting to changes in the environment through error-detecting feedback loops. Movement control by the nervous system is shaped by sensory input that enables motor commands to be adapted to the current constraints of a task. This process has been studied extensively in motor control neurophysiology by examining different forms of movement, from relatively immutable reflexes to highly variable voluntary movements. For all animals that are capable of voluntary movement, coordinated motor activity results from a combination of anticipatory and feedback control, and this integration forms the basis of movement adaptability.

Sensory information from cutaneous, proprioceptive and visual afferents is crucial for skilled grasping. The size-weight illusion powerfully demonstrates the

Corresponding author: Allan M. Smith (allan.smith@umontreal.ca). influence of visual cues in object manipulation: a small object is consistently perceived to be heavier than a larger object of the same weight due to an expected relationship between size and weight. This perception of heaviness initially influences grip force scaling [2]. As important as vision is, it is of little help in determining the friction against the fingers once the fingers have grasped an object. Consequently, this review focuses on the contribution of cutaneous afferents. It includes recent additional evidence for the fundamental and complementary roles of anticipation and feedback in the control of precision grasping that underlies the rapid adaptation to different objects in different environments. Cutaneous afferents are proposed to have several functions in the acquisition, maintenance and decay of anticipatory grip force modulation that demonstrate their role in such adaptive control. Although predictions are learned, continual cutaneous input remains crucial for skilled object manipulation.

\section{Precision grip as an experimental model for skilled movement control}

The ability to grasp an object precisely between the thumb and index finger enables humans and some other primates to perform a wide range of complex and delicate hand movements. Flexibility is an essential aspect of the dexterous manipulation of small and delicate objects, and the skilful use of tools extends the functions of the hand still further. The biomechanical advantage of an opposable thumb, combined with direct cortical control and abundant sensory feedback, enable an amazing diversity of hand movements. Lawrence and Kuypers [3] demonstrated the importance of direct connections between the cerebral cortex and the motoneurons of the hand by showing that transection of the bulbar corticospinal tract led to a loss of the ability to make relatively independent finger movements. A combination of anatomical, physiological and behavioural evidence suggests that the motor cortex, and in particular the cortex-motoneuron pathway, is essential for precise and nimble object handling [4].

In addition to this strong cortical control, the glabrous skin of the hand is densely innervated with specialized 


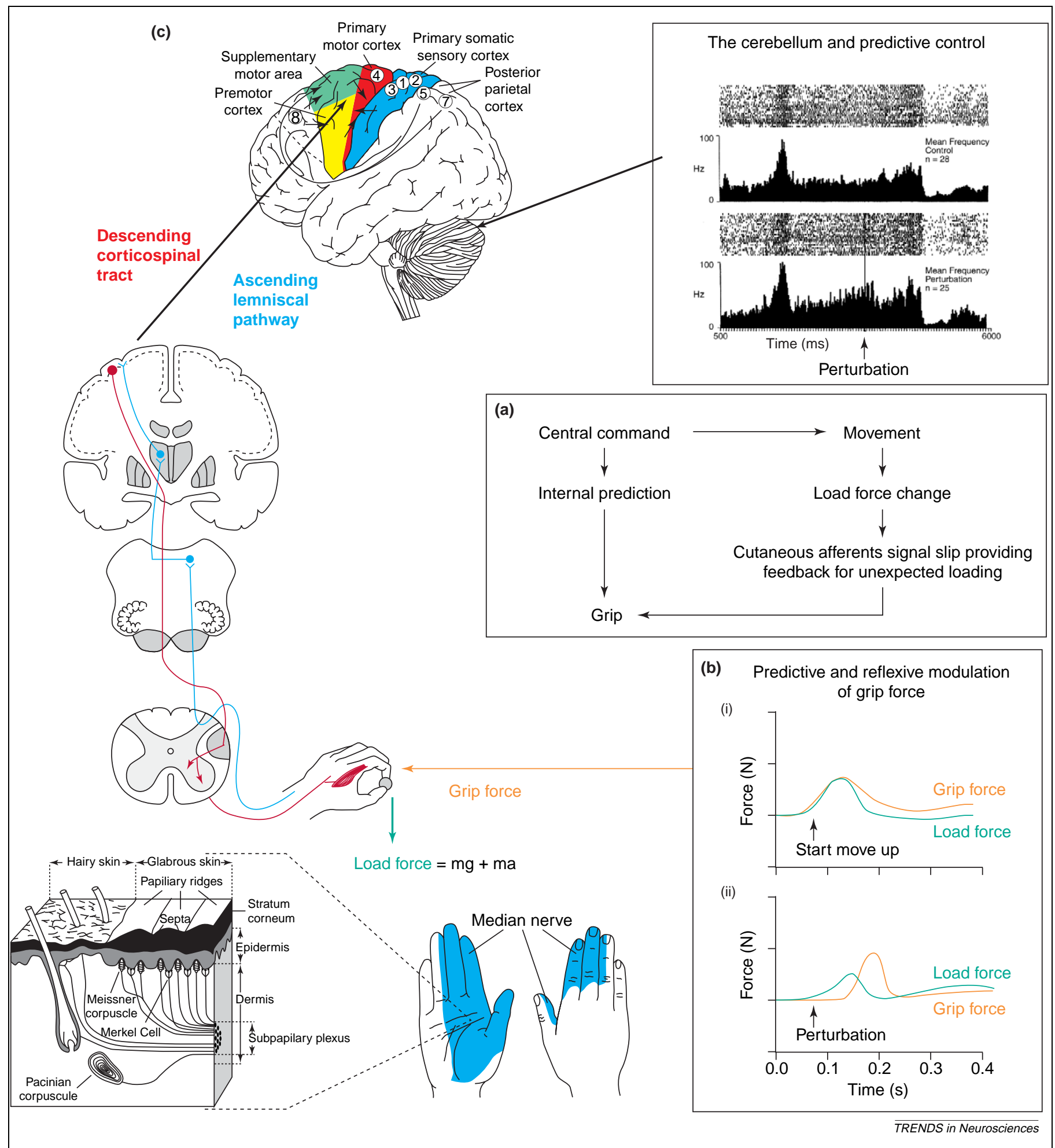

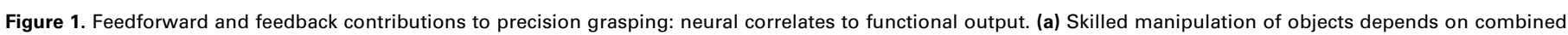

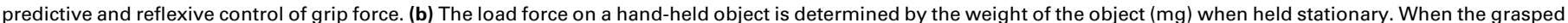

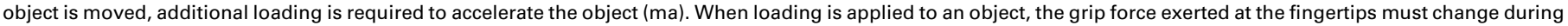

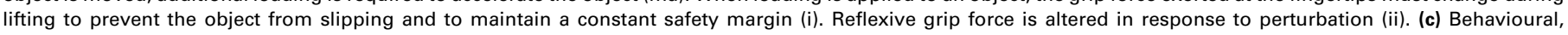
neurological-impairment and electrophysiological studies enable examination of the underlying neural control of adaptive grip force control. Several brain areas are involved

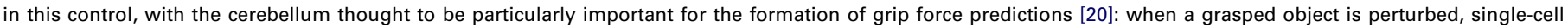

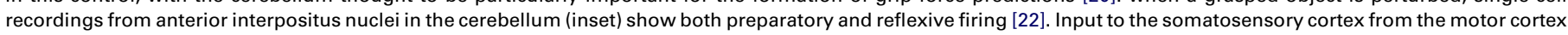

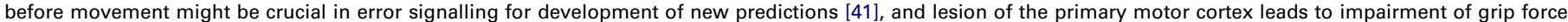

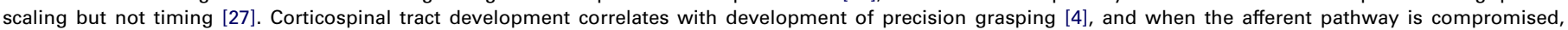

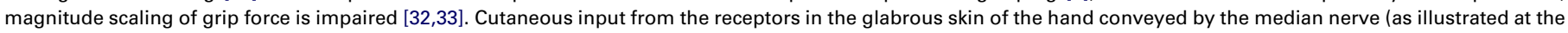
bottom of this figure) is essential for learning anticipatory grip force and also for the maintenance of accurate predictive modulation. 
cutaneous afferents that enable the adaptation of grip forces to the weight and friction of manipulated objects (Figure 1). Slowly and rapidly adapting type I afferents have been implicated in the appreciation of texture and the detection of slip, respectively [5]. These physiologically characterized afferents are thought to originate from Merkel cell complexes and Meissner corpuscles [6,7]. Recent evidence has suggested that Ruffini endings, which were previously considered to be involved in the detection of shear forces, are absent from the glabrous skin of the hand in both monkeys and humans [8,9]. Instead, configurations of Merkel cells arranged in elongated chain endings might account for the discharge pattern described as slowly adapting type II [8].

\section{Precision grip as model for predictive control}

Prediction of the consequences of a motor command is a key feature of volitional motor control [10]. An important example of such prediction is the anticipatory increase in grip force that occurs with self-initiated lifting of an object [11]. This predictive grip force response can be contrasted with a reflexive grip force modulation when the loading increase is externally generated. However, reflexive grip force modulations might be influenced by cutaneous feedback in similar ways to predictive grip force modulations [12].

In holding an object, grip force is only slightly greater than the minimum needed to prevent slip, providing a safety margin. When we move an object, the acceleration increases the tangential load force on the fingers and a parallel increase in grip force occurs with negligible delay to maintain the safety margin and to prevent slip [13]. This predictive aspect of grip force occurs in both single discrete and repetitive self-generated movements, made in different directions and at different speeds. The anticipatory modulation in grip force avoids the inevitable feedback delays associated with reflexive adjustments to changing sensory inputs. However, as will be described, behavioural data from healthy volunteers and individuals with neurological disorders show that continuous afferent feedback remains crucial for precision grasping and dexterous handling.

\section{Movement control in altered dynamics}

Prediction is central to acquiring new skills and adapting existing skills to novel environments. Predictions of the dynamics of manipulated objects are learned during development. When manipulating objects, children show an increasing ability with age to adjust grip force correctly to the load in lifting an object, with the coupling increasing towards adult values by 8 years of age [14]. The early variability in force production could reflect continual adaptation to a system that is rapidly changing, owing to, for example, increases in limb length and mass during growth.

In adults, force fields that perturb movement have been used to examine learning in the control of novel arm dynamics [15]. An important recent study used perturbing force fields to examine the learning of predictive grip force control [16]. This study showed that the acquisition of anticipatory grip-to-load force ratios preceded the acquisition of a normal arm movement trajectory. This finding suggests a separation of prediction, as evidenced by the grip force adjustments, from the control of the arm trajectory, suggesting a divergence in the rates of learning. Furthermore, it supports the proposal that both forward (for prediction) and inverse (for control) internal models contribute to voluntary movement (Box 1).

When manipulating novel objects, the CNS adapts its anticipatory grip force response to ensure predictions are accurately tuned to the dynamics of the objects. Predicting the consequences of actions on a novel object could be restricted to actions that have previously been performed on the object or, alternatively, predictive learning might generalize to novel actions. To examine the specificity of predictions about object dynamics, two experimental objects were manipulated bi-manually under computer control so that their dynamic properties could be altered on a trial-by-trial basis [17]. This enabled novel dynamic relationships between the objects and grip force control to be examined. The results indicated that predictive learning of grip force occurred only locally to the shear force directions signalled by sensory input experienced during learning. The learning of local and adaptable predictions in grip force control could underlie our ability to manipulate objects with many different force directions experienced at the fingertip. Additionally, this further highlights the importance of cutaneous input in enabling movements to be highly flexible in response to different dynamic constraints. The magnitude scaling and the timing of grip force predictions to object dynamics are discussed in Box 2.

Box 1. Internal models of the motor system that explain predictive grip force control

An important theoretical framework to explain the combination of sensory and motor signals assumes the existence of internal models of the motor system within the CNS.

When we plan an action, a transformation has to be made from sensory signals of our desired endpoint to the motor commands that will enable execution of the appropriate movement. Internal models are representations that simulate these natural transformations between sensory signals and motor commands during movement and are thought to contribute to our ability to adapt an existing skill to new environments.

A computational framework has been suggested and two main groups of internal models in the CNS, known as forward and inverse models, have been proposed [45]. The forward model represents the transformation between motor commands and sensory signals [46-48], and is the predictive model that captures the causal relationship between actions and outcome, as signalled by an efference copy of the motor command [49-51]. The inverse model simulates the transformation from sensory signals to motor commands, and therefore is essential as the controller of motor output.

Forward models have been further divided into forward dynamic models and forward sensory models, with forward dynamic models predicting future body positions after a movement and forward sensory models predicting future sensory feedback from a movement. Forward predictive models of both the motor system and the controlled object are thought to underlie our ability to perform skilled object manipulation by enabling the anticipatory modulation of grip force [20]. The adaptation of grip force prediction to novel patterns of load force could reveal many important features of forward models, and the role of cutaneous afferents in development and maintenance of these predictions. 


\section{Box 2. Timing and magnitude scaling}

The key features of anticipatory grip force modulation are the accurate prediction of the timing of the occurrence of the load force combined with an appropriate magnitude scaling. This accuracy of both timing and scaling of grip force modulation continues during motiondependent loading, supporting the presence of a forward predictive model [52]. In cyclic movements, grip and load forces are modulated simultaneously throughout [53]. The resulting oscillatory load force function is matched by an oscillatory grip force function with a strong cross-correlation between the two (Figure la). Transient loss of cutaneous feedback during cyclic loading disrupts the scaling of grip-load force coordination, although the timing or synchronization of the forces is preserved [32] (Figure lb). Similarly, after a change in gravitational field, appropriate grip-load force timing remains, but magnitude scaling requires several learning trials [54].

The behavioural dissociation between timing and amplitude of anticipatory grip adjustments $[32,55]$ points towards independent
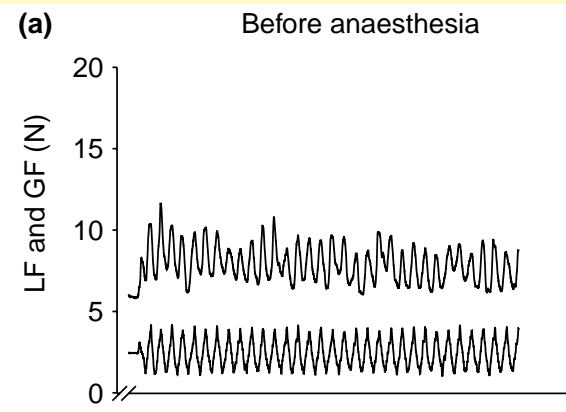

Time (s)

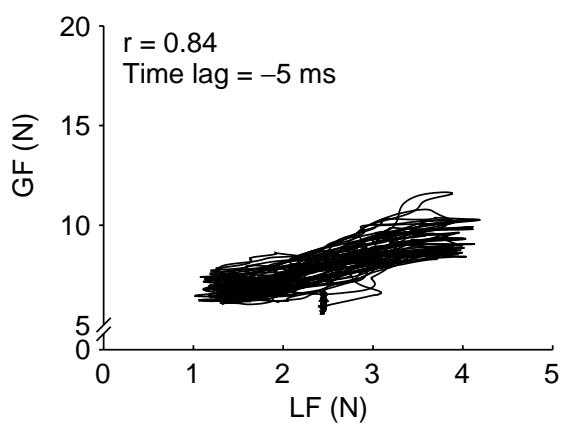

control processes for the scaling of grip force and the accuracy of timing, and implies that timing is controlled centrally but that continuous cutaneous feedback is needed to regulate the force levels themselves. This is supported by recent neural developmental evidence [56]

Previous behavioural work has shown that new timing associations between grip and load forces can be learned with repeated experience of a new timing [57]. Unlike the rapid adaptation associated with manipulating objects with different weights or frictional properties, appropriate scaling to the new timing occurred slowly, requiring $>50$ trials before the initially reactive grip force modulation became anticipatory of the new timing association and grip force magnitude decreased in amplitude. Afferent information must be crucial in driving such adaptation. Therefore timing, in contrast to magnitude scaling, is a less easily adaptable feature of the grip force response. (b)
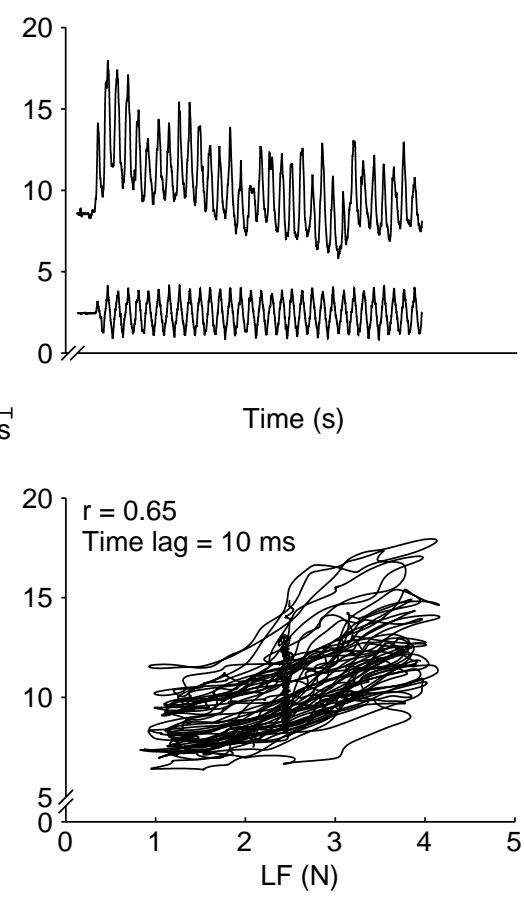

$\overline{T R E N D S}$ in Neurosciences

Figure I. Coupling of grip force and load force during a cyclic movement. An object was oscillated for $30 \mathrm{~s}$ before (a) and after (b) digital anaesthesia. Upper panels show the oscillating grip force (upper trace) and the load force (lower trace); lower panels show grip force (GF) against load force (LF). Cross-correlation ( $r$ ) shows that the temporal coupling remains between grip and load, but scaling of grip force magnitude is disrupted after anaesthesia.

\section{Neural basis for the predictive control of grip force}

Neurological impairments, functional brain imaging and single-cell recordings from behaving monkeys have all contributed to our understanding of the neural organization of grip forces during object manipulation. Comparing hand function in healthy subjects with that in neurologically impaired individuals suggests that the predictive control of grip force is achieved by several brain regions, such as the cerebellum, basal ganglia and cerebral cortex $[18,19]$. However, the individual contributions of these regions are difficult to determine because of the many interconnections between them. A key feature of grip force management during object manipulation is anticipation, and the cerebellum has long been associated with predictive control and internal models of the motor system [20]. Single-cell recordings in monkeys trained to resist perturbations of a hand-held object have indicated involvement of both the cerebellar cortex and interpositus nucleus in predictive grip force control [21,22]. Surprisingly, single-cell recordings in the primary motor cortex, supplementary motor cortex, dorsal and ventral premotor cortex, and cingulate motor area (CMA) have so far failed to find any strong evidence of activity related to the anticipation of a predictable perturbation to the hand [23-25].

Studies of neurologically impaired individuals also support the role of the cerebellum in the control of grip forces, with clear deficits in predictive control after cerebellar lesions evidenced by a lack of anticipatory temporal modulation [26]. However, predictive motor 
learning probably involves many brain areas in addition to the cerebellum, and future research will no doubt show this. Deficits in grip force control in individuals with cerebellar damage can be contrasted with deficits in individuals with hemiparesis, where anticipatory grip force control shows appropriate temporal modulation but with reduced gain [27].

\section{Cutaneous feedback is crucial in setting, maintaining and adapting the control of grip force}

If a hand-held object is very slippery, cutaneous afferents will detect slip events related to low friction and instigate not only a signal to increase grip force [28] but also a signal to reduce the acceleration of the hand to muscles at the shoulder and elbow [29]. It appears that feedback from small regions of fingertip skin can influence not only the muscles of prehension but also the muscles of the whole arm. Moreover, when called upon, these same cutaneous afferents can provide stabilizing feedback to reduce wholebody sway [30,31]. It has been postulated that the nervous system creates forward internal models of the arm and its hand-held load, to explain how we anticipate changes of inertia, friction and the centre of mass. Such anticipation has an impact not only on the grip force requirement but also on other muscles of the body (Box 1).

Cutaneous feedback can be eliminated by locally anaesthetizing the fingers to study the capacity of an acquired prediction of the inertial and frictional properties of an object to compensate for the loss of cutaneous sensation. With the fingers anaesthetized, grip force control is disrupted despite prior experience with the object; to date, there is no evidence of adaptation to this loss of cutaneous information [32,33]. This functional deterioration is probably due to an inability to predict the direction of load forces on the skin and consequent failure to adjust appropriately the opposition forces of the thumb and index finger [33]. The failure to achieve appropriate grip force modulation is compensated for by a substantial increase in both dynamic and static grip forces, regardless of the weight of the object lifted. Paradoxically, despite the compensatory increase in applied grip force, anaesthetizing the index finger and thumb reduces the maximum pinch force by $>25 \%$ [32]. There is growing evidence to suggest that cutaneous feedback provides an important tonic excitatory drive to intrinsic hand muscles. Evoked potentials in the first dorsal interosseus elicited by transcranial magnetic stimulation are significantly reduced after local anaesthesia of the median nerve. By contrast, abductor minimi $\mathrm{V}$, a muscle that is similarly innervated by the ulnar nerve but not covered by anaesthetized skin, is unaffected [34].

Taken together, these observations suggest that cutaneous feedback not only assists what has been called a 'discrete event, sensory-driven control policy' [35] but also has an equally important role in setting and maintaining the necessary tonic input required to maintain feedforward control $[32,33]$.

\section{Neural mechanism for processing cutaneous feedback}

Individuals with only moderately impaired tactile sensation caused by chronic median nerve compression at the wrist show no impairments in their ability to adapt the force ratio to various frictional conditions and no impairments in the skilful performance of precision tasks $[36,37]$. Even with severe abnormalities of median nerve conduction and discrete abnormalities of fingertip sensation, the ability to regulate grip force timing and magnitude might be conserved if some residual cutaneous feedback is present (Box 2). However, complete elimination of cutaneous feedback by local digital anaesthesia produces severe deficits in the manipulation of objects with familiar properties [33]. Individuals with polysensory neuropathy, who have a complete loss of primary afferent sensation, also show marked deficits in the control of grip forces with little or no compensation over time [38,39], reinforcing the conclusion that impairments of tactile sensation must be more severe to degrade frictional adaptation and impair the control of grip forces.

The role of sensory input in the control of grip force has been clearly demonstrated by lesions and single-cell recording studies of the somatosensory cortex (SI). Temporary inactivation of the finger area of SI produces uncoordinated grip and load forces, and an overall increase in grip force [40]. The single cell activity in SI has been examined during performance of a lift-and-hold task in monkeys, where the majority of the receptive fields in the hand area are cutaneous [41]. Rapidly adapting cells showed brief increases in activity beginning at grip onset, and slowly adapting cells had sustained activity during the stationary holding phase of the task. Both cell types responded vigorously to shear force perturbations and slip on the fingers [42]. Many of these cells showed activity increases before movement in the absence of receptive field stimulation, suggesting that, in addition to peripheral cutaneous input, these cells also receive strong excitation from movement-related regions of the brain. It is possible that these cells are tuned to the predictable elements of lifting and have a role to play if the afferent input unexpectedly departs from the normal pattern. Such responses could form the basis for constructing a forward model of object properties that would enable appropriate scaling of motor output for familiar objects [41].

\section{Concluding remarks}

Feedback from cutaneous afferents is crucial for successful feedforward control of hand-arm coordination. Recent studies provide additional evidence from behavioural, neurological impairment and electrophysiological approaches for the important contribution of tonic, continuous cutaneous afferent feedback (Figure 1). These studies demonstrate the interdependence of prediction and feedback in anticipatory grip force control, and provide a useful means of examining the role of afferent information in forward predictive models. Undoubtedly, feedback is essential for the acquisition of the internal model but more recent evidence suggests that constant uninterrupted feedback might also be necessary for maintaining previously acquired forward models.

It remains to be determined how contributions from proprioceptors and visual inputs are integrated with cutaneous feedback during object manipulation and how feedback information is integrated with prior predictions 


\section{Box 3. Questions for future research}

(i) Phasic information from cutaneous afferents has been shown to contribute to a 'discrete event, sensory-driven control policy' [35]: why is tonic input from cutaneous afferents also necessary for gripload force coordination?

(ii) Why does grip force drop rapidly as loading on an object decreases? During a cyclic movement where loading on an object rises and falls, grip force fluctuates in parallel. Grip force could be maintained at the higher level, increasing the safety margin between grip and load rather than through modulation of grip force. This characteristic could be related to the control of slip to facilitate knowledge of the dynamics of an object and so enhance skilled manipulation.

(iii) Why is self-generation of loading not sufficient for prediction to occur? In a bi-manual virtual object task, the loading between two coupled objects had to simulate an object with normal physical properties held between the hands for predictive modulation of grip force to occur [58].

in grip force control. Recent approaches to these problems propose a probabilistic framework to understand how sensory signals across different modalities are integrated [43] and how prior predictions are included with current sensory information for optimal motor commands to be issued [44]; these statistical methods could also prove useful in understanding grip force control.

The control of grip force during object manipulation provides insights into the importance of sensory afferent information that is applicable to the control of all voluntary movements. However, several questions still remain to be answered (Box 3).

\section{Acknowledgements}

We acknowledge the helpful and constructive criticism of Steven P. Wise. We also thank Claude Gauthier for help with computer graphics. A.G.W. is funded by a Wellcome Trust Advanced Training Fellowship. A.M.W. and A.G.W. were supported by the UK Medical Research Council Grant G9901257 and European Union Information Society Technologies contract IST 2001-38040. J.L.T. gratefully acknowledges a grant from Prodex, OSTC (Belgian Federal Office for Scientific, Technical and Cultural Affairs) and from the ESA, ESTEC Contract 1475/00/NL/JS. A.M.S. gratefully acknowledges the support of the CIHR and NINDS.

\section{References}

1 Lashley, K.S. (1951) The problem of serial order in behavior. In Cerebral Mechanisms in Behaviour: The Hixon Symposium (Jeffress, L.A. ed.), pp. 506-528, Wiley

2 Flanagan, J.R. and Beltzner, M.A. (2000) Independence of perceptual and sensorimotor predictions in the size-weight illusion. Nat. Neurosci. 3, 737-741

3 Lawrence, D.G. and Kuypers, H.G. (1968) The functional organization of the motor system of the monkey. Brain 91, 1-33

4 Porter, R. and Lemon, R.N. (1993) Corticospinal Function and Voluntary Movement, Clarendon Press

5 Srinivasan, M.A. et al. (1990) Tactile detection of slip: surface mircogeometry and peripheral neural codes. J. Neurophysiol. 63, 1323-1332

6 Johansson, R.S. and Vallbo, A.B. (1983) Tactile sensory coding in the glabrous skin of the human hand. Trends Neurosci. 6, 27-32

7 Johansson, R.S. and Westling, G. (1987) Signals in tactile afferents from the fingers eliciting adaptive motor-responses during precision grip. Exp. Brain Res. 66, 141-154

8 Pare, M. et al. (2002) Distribution and terminal arborizations of cutaneous mechanoreceptors in the glabrous finger pads of the monkey. J. Comp. Neurol. 455, 347-359

9 Pare, M. et al. (2003) Paucity of presumptive Ruffini corpuscles in the index finger pad of humans. J. Comp. Neurol. 456, 260-266
10 Massion, J. (1992) Movement, posture and equilibrium: interaction and coordination. Curr. Opin. Neurobiol. 38, 35-56

11 Johansson, R.S. (1996) Sensory control of dexterous manipulation in humans. In Hand and Brain: The Neurophysiology and Psychology of Hand Movements (Wing, A.M. et al., eds), pp. 381-414, Academic Press

12 Ohki, Y. and Watanabe, K. (2004) Dependence of reactive responses in human bimanual finger movements on sensory feedback and auditory cues. J. Neurophysiol. 91, 1260-1270

13 Wing, A.M. (1996) Anticipatory control of grip force in rapid arm movement. In Hand and Brain: The Neurophysiology and Psychology of Hand Movements (Wing, A.M. et al., eds), pp. 301-324, Academic Press

14 Forssberg, H. et al. (1991) Development of human precision grip-I basic coordination of force. Exp. Brain Res. 85, 451-457

15 Shadmehr, R. and Mussa-Ivaldi, F.A. (1994) Adaptive representation of dynamics during learning of a motor task. J. Neurosci. 14, 3206-3224

16 Flanagan, J.R. et al. (2003) Prediction precedes control in motor learning. Curr. Biol. 13, 146-150

17 Witney, A.G. and Wolpert, D.M. (2003) Spatial representation of predictive motor learning. J. Neurophysiol. 89, 1837-1843

18 Fellows, S.J. et al. (2001) Precision grip deficits in cerebellar disorders in man. Clin. Neurophysiol. 112, 1793-1802

19 Vaillancourt, D.E. et al. (2003) Neural basis for the processes that underlie visually-guided and internally-guided force control in humans. J. Neurophysiol. 90, 3330-3340

20 Kawato, M. (1999) Internal models for motor control and trajectory planning. Curr. Opin. Neurobiol. 9, 718-727

21 Dugas, C. and Smith, A.M. (1992) Responses of cerebellar Purkinje cells to slip of a hand-held object. J. Neurophysiol. 67, 483-495

22 Monzee, J. and Smith, A.M. (2004) Responses of cerebellar interpositus neurons to predictable perturbations applied to an object held in a precision grip. J. Neurophysiol. 91, 1230-1239

23 Boudreau, M-J. et al. (2001) Activity in ventral and dorsal premotor cortex in response to predictable force-pulse perturbations in a precision grip task. J. Neurophysiol. 86, 1067-1078

24 Cadoret, G. and Smith, A.M. (1997) Comparison of the neuronal activity in the SMA and the ventral cingulate cortex during prehension in the monkey. J. Neurophysiol. 77, 153-166

25 Picard, N. and Smith, A.M. (1992) Primary motor cortical responses to perturbations of prehension in the monkey. J. Neurophysiol. 68, 1882-1894

26 Babin-Ratte, S. et al. (1999) Impaired anticipatory finger grip-force adjustments in a case of cerebellar degeneration. Exp. Brain Res. 128, 81-85

27 Wiesendanger, M. and Serrien, D.J. (2001) Neurological problems affecting hand dexterity. Brain Res. Rev. 36, 161-168

28 Westling, G. and Johansson, R.S. (1984) Factors influencing the force control during precision grip. Exp. Brain Res. 53, 277-284

29 Saels, P. et al. (1999) Impact of surface slipperiness of grasped objects on their subsequent acceleration. Neuropsychologia 37, 751-756

30 Lackner, J.R. et al. (1999) Precision contact of the fingertip reduces postural sway of individuals with bilateral vestibular loss. Exp. Brain Res. 126, 459-466

31 Lackner, J.R. et al. (2001) Stabilization of posture by precision touch of the index finger with rigid and flexible filaments. Exp. Brain Res. 139, $454-464$

32 Augurelle, A-S. et al. (2003) Importance of cutaneous feedback in maintaining a secure grip during manipulation of hand-held objects. J. Neurophysiol. 89, 665-671

33 Monzee, J. et al. (2003) The effects of digital anaesthesia on force control using a precision grip. J. Neurophysiol. 89, 672-683

34 Rossini, P.M. et al. (1996) Focal brain stimulation in healthy humans: motor maps changes following partial hand sensory deprivation. Neurosci. Lett. 214, 191-195

35 Johansson, R.S. and Cole, K.J. (1992) Sensory-motor coordination between grasping and manipulative actions. Curr. Opin. Neurobiol. 2, $815-823$

36 Nowak, D.A. et al. (2003) Moving objects with clumsy fingers: how predictive grip force control in patients with impaired manual sensibility? Clin. Neurophysiol. 114, 472-487

37 Thonnard, J-L. et al. (1999) Effects of chronic median nerve compression at the wrist on sensation and manual skills. Exp. Brain Res. 128, 61-64 
38 Thonnard, J-L. et al. (1997) Assessment of hand function in a patient with chronic sensory demyelinating neuropathy. Neurology 49, 253-257

39 Nowak, D.A. et al. (2003) Grip force efficiency in long-term deprivation of somatosensory feedback. NeuroReport 14, 1803-1807

40 Brochier, T. et al. (1999) The effects of muscimol inactivation of small regions of motor and somatosensory cortex on independent finger movements and force control in the precision grip. Exp. Brain Res. $128,31-40$

41 Salimi, I. et al. (1999) Neuronal activity in somatosensory cortex of monkeys using a precision grip. I Receptive fields and discharge patterns. J. Neurophysiol. 81, 825-834

42 Salimi, I. et al. (1999) Neuronal activity in somatosensory cortex of monkeys using a precision grip task. II Responses to object texture and weights. J. Neurophysiol. 81, 835-844

43 Ernst, M.O. and Banks, M.S. (2002) Humans integrate visual and haptic information in a statistically optimal fashion. Nature 415, 429-433

44 Kording, K.P. and Wolpert, D.M. (2004) Bayesian integration in sensorimotor learning. Nature 427, 245-247

45 Wolpert, D.M. and Ghahramani, Z. (2000) Computational principles of movement neuroscience. Nat. Neurosci. 3, 1212-1217

46 Kawato, M. et al. (1987) A hierarchical neural network model for the control and learning of voluntary movements. Biol. Cybern. 56, 1-17

47 Jordan, M.I. and Rumelhart, D.E. (1992) Forward models: supervised learning with a distal teacher. Cogn. Sci. 16, 307-364

48 Miall, R.C. and Wolpert, D.M. (1996) Forward models for physiological motor control. Neural Netw. 9, 1265-1279
49 Sperry, R.W. (1950) Neural basis of the spontaneous optokinetic response produced by visual inversion. J. Comp. Physiol. Psychol. 43, $482-489$

50 Von Holst, E. (1954) Relations between the central nervous systems and the peripheral organ. Br. J. Anim. Behav. 2, 89-94

51 Jeannerod, M. et al. (1979) Corollory discharge: its possible implications in visual and occulomotor interactions. Neuropsychologia 17, 241-258

52 Flanagan, J.R. and Wing, A.M. (1993) Modulation of grip force with load force during point-to-point arm movements. Exp. Brain Res. 95, 131-143

53 Flanagan, J.R. and Wing, A.M. (1995) The stability of precision grip forces during cyclic arm movements with a hand-held load. Exp. Brain Res. 105, 455-464

54 Augurelle, A-S. et al. (2003) The effects of a change in gravity on the dynamics of prehension. Exp. Brain Res. 148, 533-540

55 Nowak, D.A. et al. (2001) The effects of digital anaesthesia on predictive grip force adjustments during vertical movements of a grasped object. Eur. J. Neurosci. 14, 756-762

56 Blank, R. et al. (2001) Human development of grip force modulation to cyclic movement-induced inertial loads. Exp. Brain Res. 138, 193-199

57 Witney, A.G. et al. (1999) Predictive motor learning of temporal delays. J. Neurophysiol. 82, 2039-2048

58 Blakemore, S.J. et al. (1998) Predicting the consequences of our own actions: the role of sensorimotor context estimation. J. Neurosci. 18, $7511-7518$

\section{Free journals for developing countries}

The WHO and six medical journal publishers have launched the Access to Research Initiative, which enables nearly 70 of the world's poorest countries to gain free access to biomedical literature through the Internet.

The science publishers, Blackwell, Elsevier, the Harcourt Worldwide STM group, Wolters Kluwer International Health and Science, Springer-Verlag and John Wiley, were approached by the WHO and the British Medical Journal in 2001. Initially, more than 1000 journals will be available for free or at significantly reduced prices to universities, medical schools, research and public institutions in developing countries. The second stage involves extending this initiative to institutions in other countries.

Gro Harlem Brundtland, director-general for the WHO, said that this initiative was 'perhaps the biggest step ever taken towards reducing the health information gap between rich and poor countries'. 